\title{
Global extracellular vesicle proteomic signature defines U87-MG glioma cell hypoxic status with potential implications for non-invasive diagnostics
}

\author{
Vineesh Indira Chandran ${ }^{1}$ (D . Charlotte Welinder ${ }^{1} \cdot$ Kelin Gonçalves de Oliveira $^{1}$ - Myriam Cerezo-Magaña ${ }^{1}$. \\ Ann-Sofie Månsson ${ }^{1} \cdot$ Maria C. Johansson ${ }^{1} \cdot$ Gyorgy Marko-Varga ${ }^{2} \cdot$ Mattias Belting $^{1,3,4}$
}

Received: 26 April 2019 / Accepted: 6 August 2019 / Published online: 14 August 2019

(c) The Author(s) 2019

\begin{abstract}
Purpose Glioblastoma multiforme (GBM) is the most common and lethal of primary malignant brain tumors. Hypoxia constitutes a major determining factor for the poor prognosis of high-grade glioma patients, and is known to contribute to the development of treatment resistance. Therefore, new strategies to comprehensively profile and monitor the hypoxic status of gliomas are of high clinical relevance. Here, we have explored how the proteome of secreted extracellular vesicles (EVs) at the global level may reflect hypoxic glioma cells.

Methods We have employed shotgun proteomics and label free quantification to profile EVs isolated from human highgrade glioma U87-MG cells cultured at normoxia or hypoxia. Parallel reaction monitoring was used to quantify the identified, hypoxia-associated EV proteins. To determine the potential biological significance of hypoxia-associated proteins, the cumulative $Z$ score of identified EV proteins was compared with GBM subtypes from HGCC and TCGA databases.

Results In total, 2928 proteins were identified in EVs, out of which 1654 proteins overlapped with the ExoCarta EV-specific database. We found 1034 proteins in EVs that were unique to the hypoxic status of U87-MG cells. We subsequently identi-

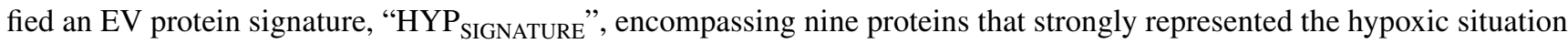
and exhibited close proximity to the mesenchymal GBM subtype.

Conclusions We propose, for the first time, an EV protein signature that could comprehensively reflect the hypoxic status of high-grade glioma cells. The presented data provide proof-of-concept for targeted proteomic profiling of glioma derived EVs, which should motivate future studies exploring its utility in non-invasive diagnosis and monitoring of brain tumor patients.
\end{abstract}

Keywords Glioblastoma $\cdot$ Hypoxia $\cdot$ Extracellular vesicles $\cdot$ Mass spectrometry $\cdot$ Label free quantification

\section{Introduction}

Electronic supplementary material The online version of this article (https://doi.org/10.1007/s11060-019-03262-4) contains supplementary material, which is available to authorized users.

Vineesh Indira Chandran

vineeshindirachandran@gmail.com

1 Department of Clinical Sciences, Lund, Division of Oncology and Pathology, Lund University, Lund, Sweden

2 Department of Biomedical Engineering, Clinical Protein Science \& Imaging, Biomedical Center, Lund University, Lund, Sweden

3 Department of Hematology, Oncology and Radiophysics, Skåne University Hospital, Lund, Sweden

4 Department of Immunology, Genetics and Pathology, Uppsala University, Uppsala, Sweden
Glioblastoma multiforme (GBM) is the most common and malignant type of primary brain tumor in adults with a median survival of approximately 15 months [1-3]. GBM is identified from less malignant, low grade gliomas, by extensive regions of hypoxia [4] that directly correlate with the aggressive behaviour [5]. Hypoxia results from the high proliferative and metabolic activity of malignant cells [6] and is associated with pseudopalisading necrosis as well as vascular hyperproliferation [7]. Tumor hypoxia modulates stromal cell interactions in the microenvironment that further support the survival and dissemination of malignant cells [4, 8-11]. Numerous studies have previously shown that tumor progression is driven by hypoxic signaling [12], and the expression of hypoxia-related markers correlate 
with poor patient outcome in several tumor types, including GBM [13]. However, the development of strategies for non-invasive monitoring of brain tumor hypoxic signalling remains a challenge of high clinical relevance, especially with regard to the relative inaccessibility and spatiotemporal heterogeneity of GBM tumors.

Extracellular vesicles (EVs) are excessively secreted by tumor cells into the circulation, and are emerging as a promising candidate for liquid biopsy-based approaches in cancer [14-16]. Exosomes and microvesicles are lipid-bilayer EVs [17] that have come to be recognized in intercellular communication, promoting the development and progression of various disease conditions [18]. Numerous studies have shown that exosome-like EVs may mediate hypoxiadependent intercellular signaling in GBM [19]. Moreover, pilot studies based on an antibody array targeted at angiogenesis-related proteins, suggested that the EV proteome may reflect the tumor oxygenation status in GBM [20]. To further develop EV-based strategies for non-invasive tumor diagnosis and monitoring of hypoxia, it is essential to comprehensively identify proteins that are efficiently sorted to EVs and that reflect the hypoxic status of the cell or tissue of origin.

In this study, we employed label free quantification (nontargeted method) and parallel reaction monitoring (targeted method) to globally characterize the proteome of EVs derived from U87-MG high-grade glioma cells with the aim to understand how EV profiling can be exploited to noninvasively define the hypoxic status of glioma tumors.

\section{Results}

\section{Global proteome identification in EVs derived from high-grade glioma cells}

EVs from U87-MG, i.e. the most well-characterized human glioma cell-line $[21,22]$, grown under normoxic $\left(\mathrm{EV}_{\text {NORM }}\right)$ or hypoxic $\left(\mathrm{EV}_{\mathrm{HYP}}\right)$ conditions were isolated by standard sequential ultracentrifugation [20]. The size distribution and morphology of EVs was analyzed by transmission electron microscopy (TEM), where $\mathrm{EV}_{\mathrm{NORM}}$ and $\mathrm{EV}_{\mathrm{HYP}}$ predominantly were found in the size range of $50-150 \mathrm{~nm}$ in diameter with no apparent difference in their morphology (Fig. 1a, b). Nanoparticle tracking analysis (NTA) showed similar size distribution, where both $\mathrm{EV}_{\mathrm{NORM}}$ and $\mathrm{EV}_{\mathrm{HYP}}$ were found in the size range of $80-150 \mathrm{~nm}$ (Fig. 1c, d), which is consistent with the typical size distribution profile of exosomes [23]. We found significantly increased secretion of EVs by U87-MG cells when cultured under hypoxia as compared to normoxia (Fig. 1d), which is in accordance with previous findings $[24,25]$. Currently, in addition to the mechanism of biogenesis and size [26], EVs are generally referred to as exosomes also based on the expression of CD9, CD63, and CD81 proteins [27], which were all found to be present in U87-MG derived EVs, together with a strong enrichment of the membrane raft marker Flotillin 1 (Fig. 1e).

We then employed shotgun proteomics by data-dependent acquisition to comprehensively determine the proteome of $\mathrm{EV}_{\text {NORM }}$ and $\mathrm{EV}_{\text {HYP }}$ derived from U87-MG cells. We identified a total of $2089 \mathrm{EV}_{\mathrm{HYP}}$ and $2035 \mathrm{EV}_{\text {NORM }}$ proteins (Fig. 1f; Supplementary Tables 1, 2). There were 1034 protein groups unique to $\mathrm{EV}_{\mathrm{HYP}}$ (Fig. 1f; Supplementary Table 3 ) and 1055 protein groups common to both $\mathrm{EV}_{\text {NORM }}$ and $\mathrm{EV}_{\mathrm{HYP}}$ (Fig. 1f; Supplementary Table 4). We next created a multiconsensus list combining $\mathrm{EV}_{\mathrm{NORM}}$ and $\mathrm{EV}_{\mathrm{HYP}}$ protein identities (Supplementary Table 5) and then compared the multiconsensus protein group to the ExoCarta EV public database [28]. The multiconsensus EV identities (2928 proteins) showed extensive overlapping of 1654 common identities with the ExoCarta database and also identified 1274 unique identities (Fig. 1g), which support the sensitivity of detection of the EV proteome with the current approach.

\section{Processing of the EV proteome by label free quantification (LFQ)}

Discovery MS analysis resulted in the identification of thousands of proteins, and it is not feasible to analyze the abundance signature of each individual protein by targeted MS/ MS. Therefore, to filter the proteins identified in $\mathrm{EV}_{\text {NORM }}$ and $\mathrm{EV}_{\mathrm{HYP}}$ based on their significance in hypoxia, we subjected the discovery MS-identified proteins to nontargeted LFQ in Proteome Discoverer (PD) version 2.2 (Fig. 2a). We could then obtain the abundance value of each protein in $\mathrm{EV}_{\text {HYP }}$ and $\mathrm{EV}_{\text {NORM }}$ in terms of the LC/MS precursor peak quantification of the unique peptides for a particular protein. Subsequently, a ratio of the abundance values of each protein in $\mathrm{EV}_{\mathrm{HYP}}$ over $\mathrm{EV}_{\text {NORM }}$ was calculated, which identified a total of 580 hypoxia significant $\left(\mathrm{H}_{\text {significant }}\right)$ proteins (Log2 fold change, cut-off $>0.01$ ), and other proteins that were above $\log 2$ fold change cut-off $>0.01$, were taken as hypoxia downregulated $\left(\mathrm{H}_{\text {nonsignificant }}\right.$ ) proteins (Supplementary Table 6).

$\mathrm{H}_{\text {significant }}$ proteins were found to be distributed mostly in nucleic acid binding, hydrolase, enzyme modulators, and cytoskeletal protein subclasses, as determined by Gene Ontology system of classification using PANTHER version 14.0 [29] (Fig. 2b). Then we analysed the differences in functional classification pertaining to biological processes, molecular functions, and cellular localization of the $\mathrm{H}_{\text {significant }}$ (orange bars) and $\mathrm{H}_{\text {nonsignificant }}$ (blue bars) proteins (Fig. 2). A substantially higher number of $\mathrm{H}_{\text {significant }}$ 
Fig. 1 Characterization of EVs isolated from normoxic and hypoxic glioma cells. Electron microscopy shows comparable shape and size distribution of EVs isolated from normoxic (a) and hypoxic (b) U87-MG cells. Scale bar, $200 \mathrm{~nm}$. Nano tracking analysis showing variations in particle concentration in normoxia (c) and hypoxia (d) of U87-MG cell-derived EVs. e U87-MG cells and EVs were probed for $\mathrm{CD} 9, \mathrm{CD} 63$, CD81, Flotillin 1, and tubulin by immunoblotting. f Venn diagram illustrating protein groups identified in normoxic $(n=9)$ and hypoxic $(n=12)$ cell EVs using LC-MS/MS procedures, as indicated in the Supplementary Methods section. g Venn diagram showing comparison of protein groups identified in U87-MG cell EVs by LC-MS/ MS with ExoCarta, a public EV proteomics database
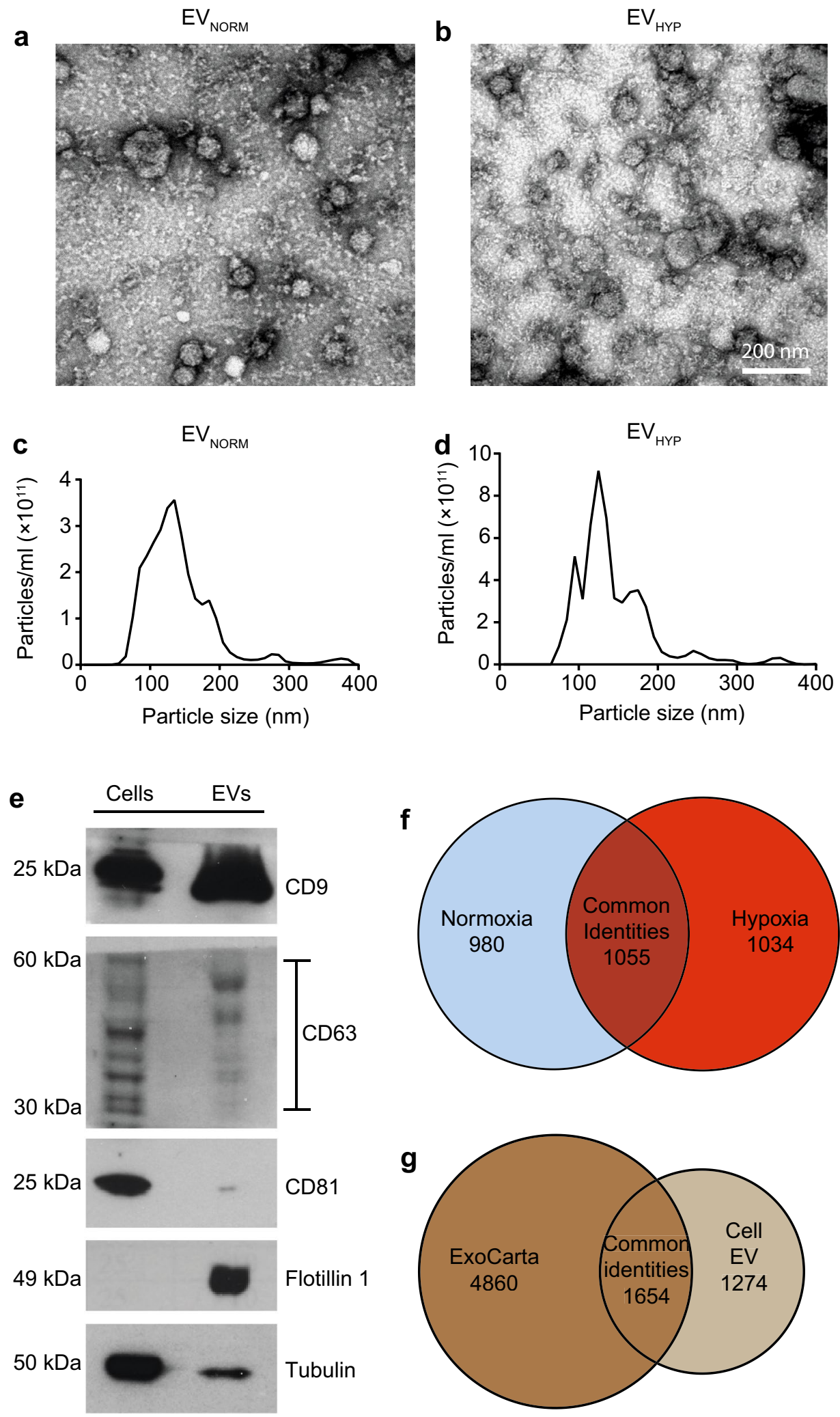

proteins were localized in organelles (GO:0,043,226) and macromolecular complexes (GO:0,032,991) compared to $\mathrm{H}_{\text {nonsignificant }}$ proteins (Fig. 2c). More $\mathrm{H}_{\text {significant }}$ proteins were associated with cellular (GO:0,009,987), metabolic
(GO:0,008,152), and cellular component biogenesis processes (GO:0,071,840) and catalytic activity (GO:0,003,824) compared to $\mathrm{H}_{\text {nonsignificant }}$ proteins (Fig. 2d, e), consistent with characteristics of the hypoxic tumor state $[30,31]$. 

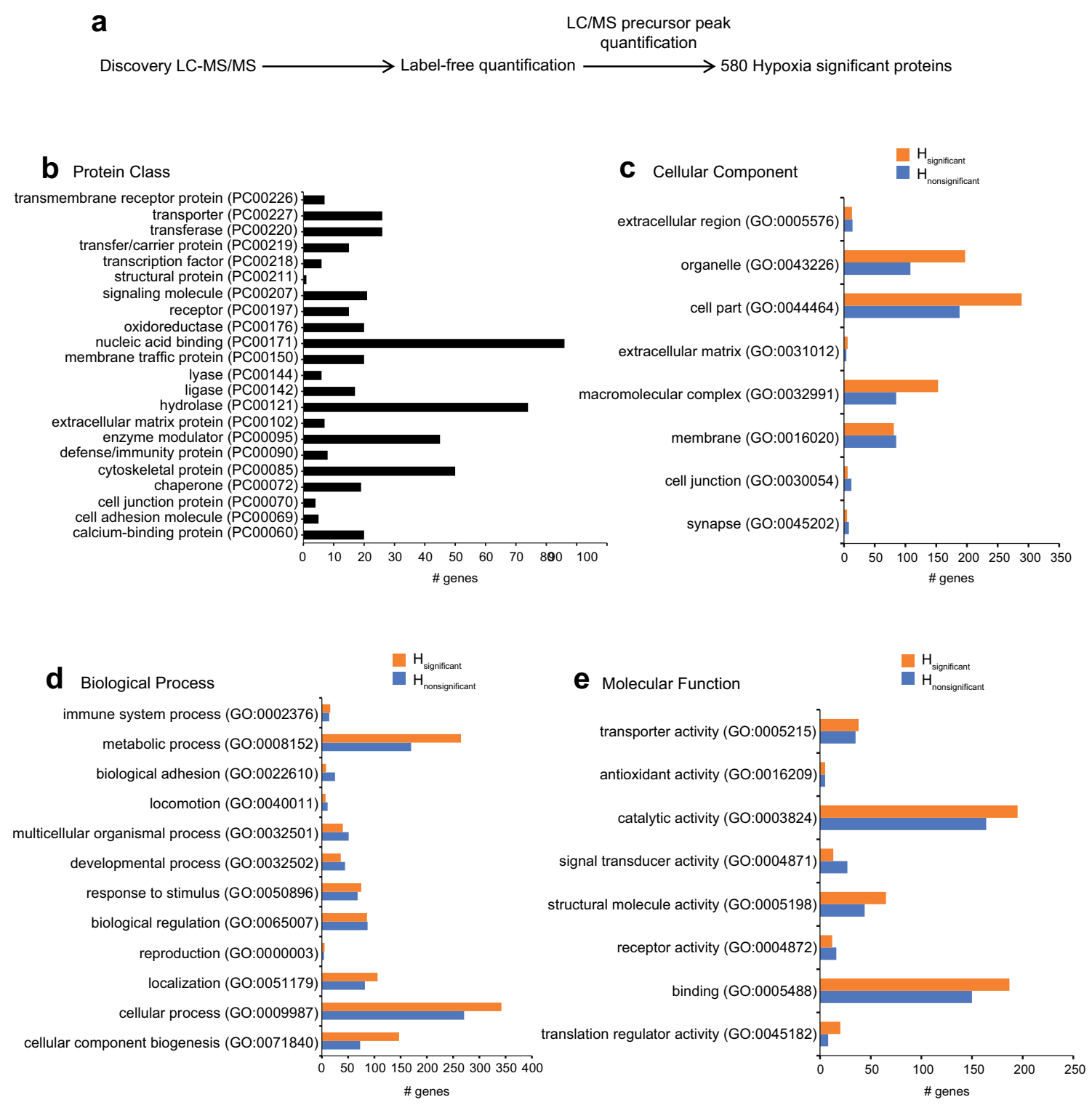

Fig. 2 Non-targeted label free quantification of EV protein groups identified by LC-MS/MS. Schematic diagram showing label free quantification of EV proteins identified by Proteome Discoverer (a).

\section{Validation of $\mathrm{H}_{\text {significant }}$ profile by parallel reaction monitoring (PRM)}

To validate the $\mathrm{H}_{\text {significant }}$ proteins identified above by LFQ, we next performed PRM (Fig. 3a). A set of selection criteria specific for targeted PRM analysis as described in Rauniyar was applied [32], including peptide length, uniqueness, miscleavage, modification, precursor charge, chromatographic peak, and signal intensity to further filter identified protein groups and select appropriate quantotypic peptides for proteins of interest using Skyline version 3.1. In addition, we added a few protein groups based on their relevance in glioma. Consequently, we selected a total of 135 protein
Functional classification analyses assigned as protein class (b), cellular component (c), biological process (d), and molecular function (e) of $\mathrm{H}_{\text {significant }}$ (orange bars) and $\mathrm{H}_{\text {nonsignificant }}$ (blue bars) proteins

groups with 5 unique quantotypic peptides per protein group for quantification by targeted PRM. Firstly, we performed an unscheduled PRM run on $\mathrm{EV}_{\text {NORM }}$ and $\mathrm{EV}_{\mathrm{HYP}}$ samples to analyze the ionization of selected peptides and optimize their retention time and transition charge state. The chromatogram output was analyzed in Skyline and the 2 to 3 most quantotypic flyable peptides and appropriate transition states per protein were selected for the scheduled PRM run for all 135 protein groups (Supplementary Table 7).

The peak normalized areas (PAN) of individual peptides of all proteins analyzed were extracted from the Skyline, and the average of replicate PAN values of each individual peptide of all proteins in $\mathrm{EV}_{\mathrm{NORM}}$ or $\mathrm{EV}_{\mathrm{HYP}}$ 
a Parallel reaction monitoring

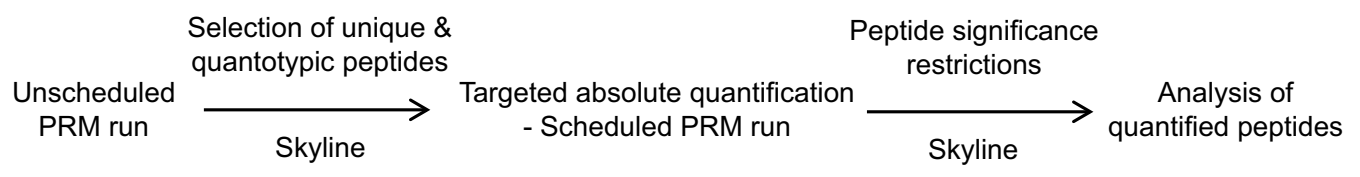

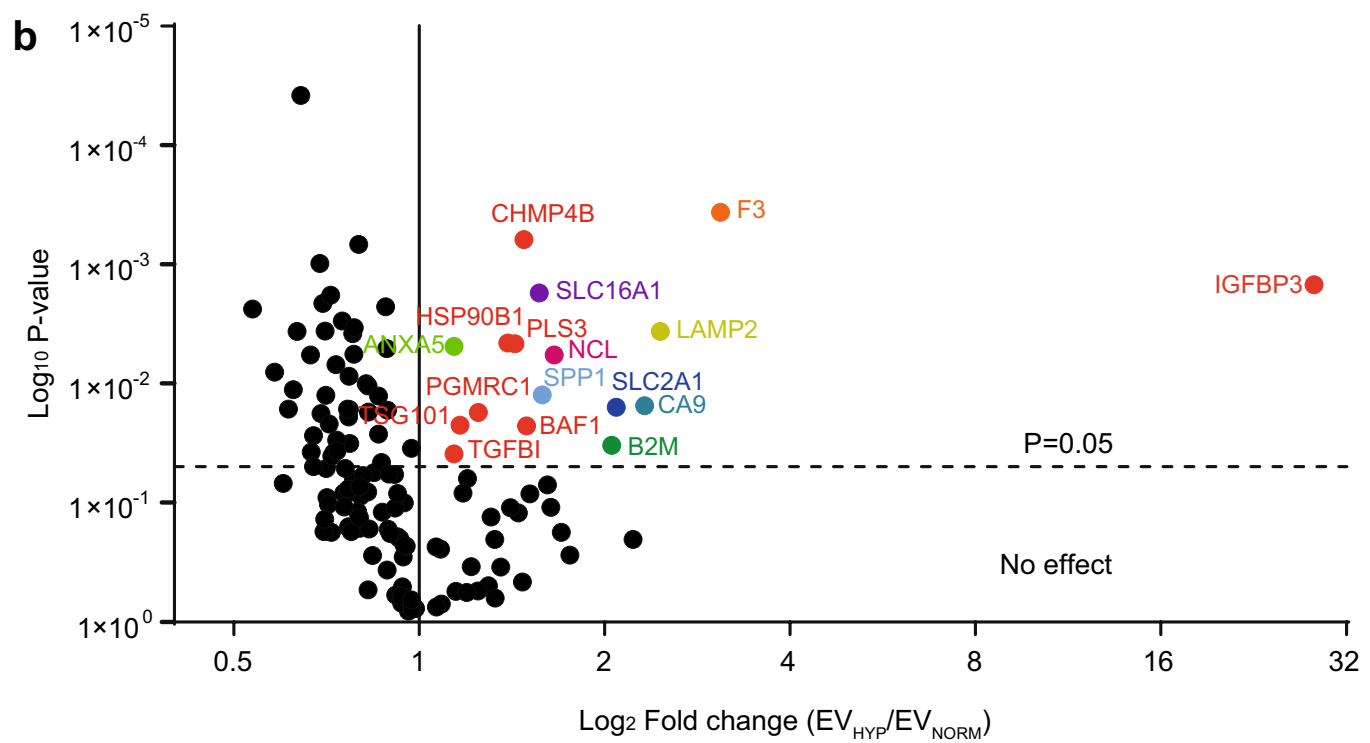

\begin{tabular}{c|c|c|c|}
\hline C & Description & Fold change & P-value \\
\hline P17936 & Insulin-like growth factor-binding protein 3 (IGFBP3) & 28.36 & 0.0015 \\
\hline P13726 & Tissue factor (F3) & 3.08 & 0.0004 \\
\hline Q16790 & Carbonic anhydrase 9 (CA9) & 2.32 & 0.0153 \\
\hline P11166 & Solute carrier family 2, facilitated glucose transporter 1 (SLC2A1) & 2.09 & 0.0157 \\
\hline P19338 & Nucleolin (NCL) & 1.66 & 0.0057 \\
\hline P10451-5 & Osteopontin (SPP1) & 1.58 & 0.0124 \\
\hline P53985 & Monocarboxylate transporter 1 (SLC16A1) & 1.56 & 0.0017 \\
\hline O00264 & Membrane-associated progesterone receptor component 1 (PGRMC1) & 1.25 & 0.0174 \\
\hline P08758 & Annexin 5 (ANXA5) & 1.14 & 0.0048 \\
\hline
\end{tabular}

Fig. 3 Relative quantification of selected EV protein groups/peptides by LC-PRM-MS/MS. a Schematic diagram showing quantification of proteome extracted from normoxic or hypoxic U87-MG cell-derived EVs by PRM. b Volcano plot showing differentially expressed proteins in EVs isolated from normoxic and hypoxic U87-MG cells. Each protein is represented as a dot and is mapped according to its

samples were calculated. In all cases, the selected peptides of 135 candidate proteins had quantifiable distribution of area under curve for the identified peptide transitions (Supplementary Table 8). On analysing the fold change, we found 17 proteins significantly differentially expressed in $\mathrm{EV}_{\text {HYP }}$ as compared to $\mathrm{EV}_{\text {NORM }}$ (Fig. 3b; Supplementary Table 8). We further applied peptide significance and normalized peak area restrictions on the hypoxia response of the $\mathrm{H}_{\text {significant }} \mathrm{EV}$ proteins $(N=17)$ and filtered it down to a signature of 9 proteins that included Insulin-like Growth Factor-Binding Protein 3 (IGFBP3), Tissue Factor (F3), fold change $\left(\mathrm{EV}_{\mathrm{HYP}}\right.$ compared to $\left.\mathrm{EV}_{\mathrm{NORM}}\right)$ on the abscissa axis $(\mathrm{x})$ and $t$ test $P$ value on the ordinate axis (y). Colored dots indicate significant proteins and black dots indicate non-significant proteins. c List of proteins found enriched (fold change) in EVs isolated from hypoxic as compared with normoxic U87-MG cells, as quantified by PRM

Carbonic Anhydrase 9 (CA9), Solute Carrier Family 2 Facilitated Glucose Transporter Member 1 (SLC2A1), Nucleolin (NCL), Osteopontin (SPP1), Monocarboxylate Transporter 1 (SLC16A1), Membrane-Associated Progesterone Receptor Component 1 (PGRMC1), and Annexin A5 (ANXA5) (Fig. 3c). These proteins defined a profile of unique proteins $(N=9)$ efficiently sorted from donor cells to EVs and enriched at hypoxic conditions, hereafter referred to as "HYP ${ }_{\text {SIGNATURE" (the PAN of the replicates }}$ of the different peptides is given in Supplementary Fig. 1). 


\section{HYP $_{\text {SIGNATURE }}$ can identify GBM mesenchymal subtype}

We assayed the pathways enriched by the HYP SIGNATURE $_{\text {inte }}$ proteins using ConsensusPathDB-human interaction database [33]. This identified $\mathrm{HYP}_{\text {SIGNATURE }}$ to be closely associated with the Hypoxia-Inducible Factor- $1 \alpha(\mathrm{HIF}-1 \alpha)$ transcription factor network (adjusted $P$ value $=0.00012$ ) and HIF-1 signalling pathway (adjusted $P$ value $=0.0057$ ) with high significance (Fig. 4a). Tissue factor (F3) was previously shown by our group to be enriched in hypoxia-derived EVs
[20]. The hypoxic enrichment of other top candidates of the HYP $_{\text {SIGNATURE }}$ (Fig. 3c), was supported by immunoblotting, which showed increased levels of IGFBP3 (Fig. 4b) and CA9 (Fig. 4c). Immunoblotting analysis was unable to detect other candidate proteins (NCL, SLC16A1, SPP1, ANXA5) in EVs, either from normoxia or hypoxia (Supplementary Fig. 2b). A potential limitation of these results is the lack of EV housekeeping proteins, and equal protein loading rely on BCA total protein concentration. However, gene array analysis showed increased expression of IGFBP3 $(P=0.0012), \mathrm{F} 3$

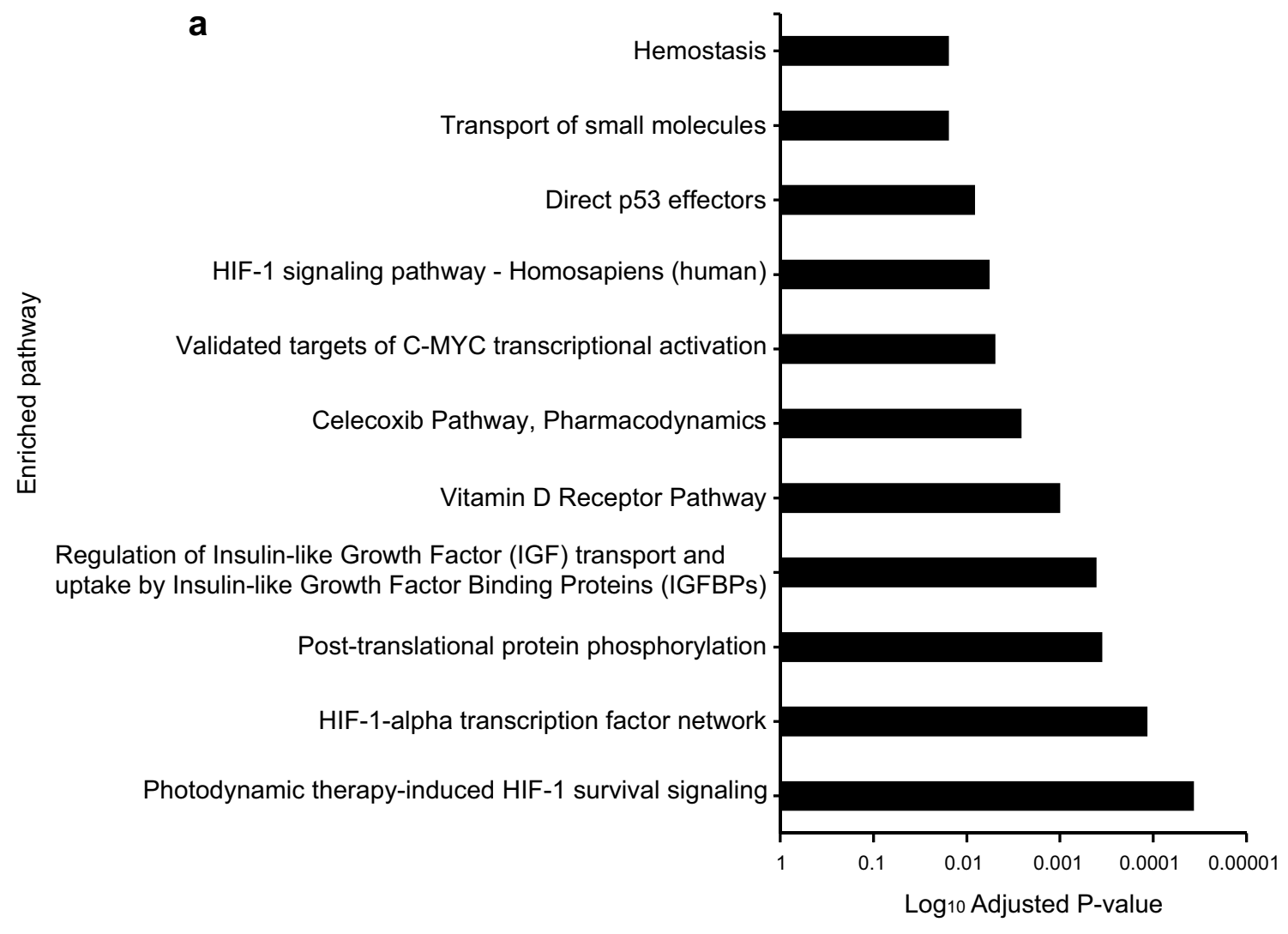

b

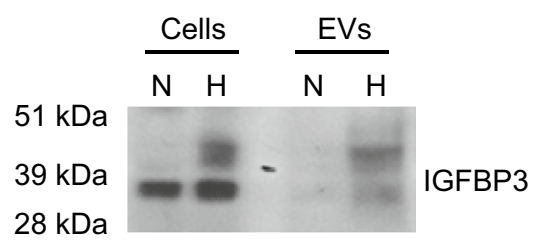

C

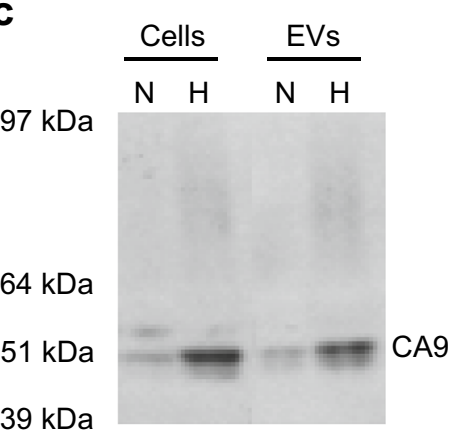

Fig. 4 Validation of $\mathrm{HYP}_{\text {SIGNATURE }}$ top candidate proteins and association with HIF-dependent pathways. a Pathway enrichment of HYP $_{\text {SIGNATURE }}$ proteins analyzed by ConsensusPathDB-human inter- action database. Immunoblotting showing enrichment of IGFBP3 (b) and $\mathrm{CA} 9$ (c) in $\mathrm{EV}_{\mathrm{HYP}}$ as compared with $\mathrm{EV}_{\mathrm{NORM}}$ samples 
$(P=0.0001)$, CA9 $(P=0.0001)$, SLC2A1 $(P=0.0001)$, and PGRMC1 $(P=0.0017)$ mRNA in hypoxic as compared with normoxic U87-MG cells (Supplementary Fig. 2a).

Several studies have established the association of GBM mesenchymal subtype with hypoxia and an aggressive tumor phenotype [34-36]. To address how the HYP ${ }_{\text {SIGNATURE }}$ may associate with the mesenchymal phenotype, we compared the cumulative $Z$ score of HYP $_{\text {SIGNATURE }}$ with different subtypes of primary GBM cells obtained from Human Glioblastoma Cell Culture (HGCC) i.e. classical, proneural, neural and mesenchymal
(Fig. 5a). The cumulative $\mathrm{HYP}_{\text {SIGNATURE }} Z$ score (1.78) was in close proximity to the HGCC mesenchymal subtype (0.24), evident by their average positive $Z$ score as compared with the classical $(-0.18)$, proneural $(-0.28)$, and neural (-0.41) subtypes (Fig. 5b). Next, we compared

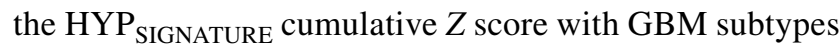
obtained from Cancer Genome Atlas Program (TCGA) using the Gliovis portal, which again showed the proximity of HYP $_{\text {SIGNATURE }} Z$ score with the mesenchymal (1.26) as compared with classical (0.94), proneural (0.89), and neural (0.83) GBM subtypes (Fig. 5c).

a

\begin{tabular}{|c|c|c|c|c|c|c|c|c|c|c|}
\hline \multirow[b]{2}{*}{ Uniprot ID } & \multirow[b]{2}{*}{ HGNC symbol } & \multicolumn{9}{|c|}{ Z-score values } \\
\hline & & $\mathrm{EV}_{\mathrm{HYP}}$ & \multirow[b]{2}{*}{ MS } & \multicolumn{2}{|c|}{ HGCC } & \multirow[b]{2}{*}{ NL } & \multicolumn{4}{|c|}{ TCGA } \\
\hline \multirow[b]{2}{*}{ P17936 } & \multirow[b]{2}{*}{ IGFBP3 } & & & $C L$ & PN & & MS & $\mathrm{CL}$ & PN & NL \\
\hline & & 1.94 & 0.50 & -0.50 & -0.24 & -1.26 & 1.74 & 1.37 & 1.15 & 1.19 \\
\hline P13726 & F3 & 1.60 & 0.02 & 0.41 & -0.57 & -0.10 & 1.27 & 1.49 & 0.55 & 1.12 \\
\hline Q16790 & CA9 & 1.66 & 0.25 & -0.36 & -0.09 & -0.27 & 3.20 & 2.37 & 2.86 & 2.35 \\
\hline P08758 & ANXA5 & 1.72 & 0.45 & -0.23 & -0.79 & -0.35 & 1.85 & 2.31 & 1.05 & 1.85 \\
\hline P10451-5 & SPP1 & 1.15 & 0.47 & -0.49 & -0.34 & -0.82 & 1.98 & 1.17 & 1.42 & 1.91 \\
\hline P19338 & $\mathrm{NCL}$ & 1.10 & -0.13 & 0.45 & -0.21 & -0.15 & -1.51 & -1.30 & -1.30 & -3.10 \\
\hline P53985 & SLC16A1 & 2.17 & 0.18 & 0.09 & -0.46 & -0.39 & 1.23 & 0.87 & 0.95 & 0.71 \\
\hline 000264 & SLC2A1 & 1.83 & 0.63 & -0.58 & -0.63 & -0.83 & 1.14 & 0.64 & 0.82 & 0.56 \\
\hline P11166 & PGRMC1 & 2.90 & -0.18 & -0.40 & 0.83 & 0.53 & 0.54 & -0.40 & 0.96 & 0.86 \\
\hline
\end{tabular}

b

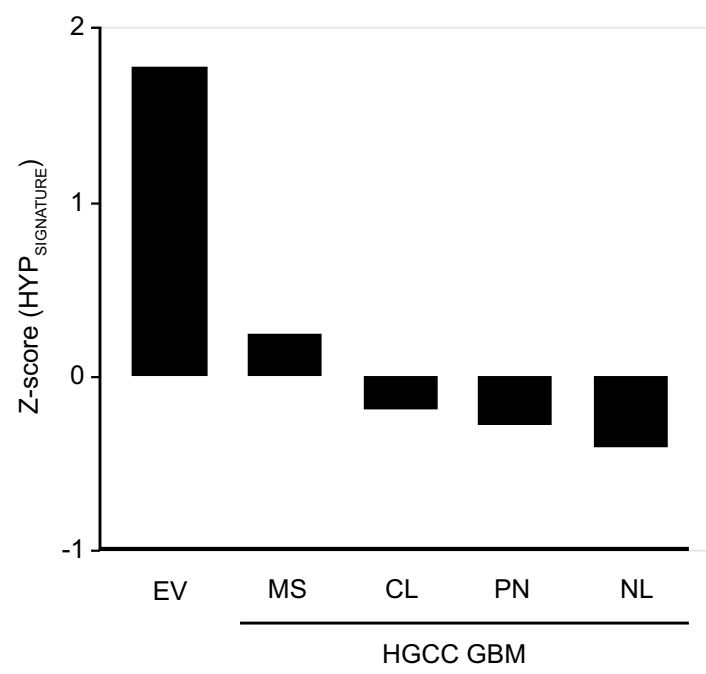

Fig. 5 HYP $_{\text {SIGNATURE }}$ can identify GBM mesenchymal subtype (a) Table showing $Z$ score values of $\mathrm{HYP}_{\text {SIGNATURE }}$ candidates obtained from U87-MG cell EVs, HGCC, and TCGA database. For U87-MG cell EVs, corresponding $\mathrm{HYP}_{\text {SIGNATURE }}$ candidate protein values from normoxia-derived EVs were used as a reference; for TCGA GBM subtypes, low grade glioma gene expression data of the

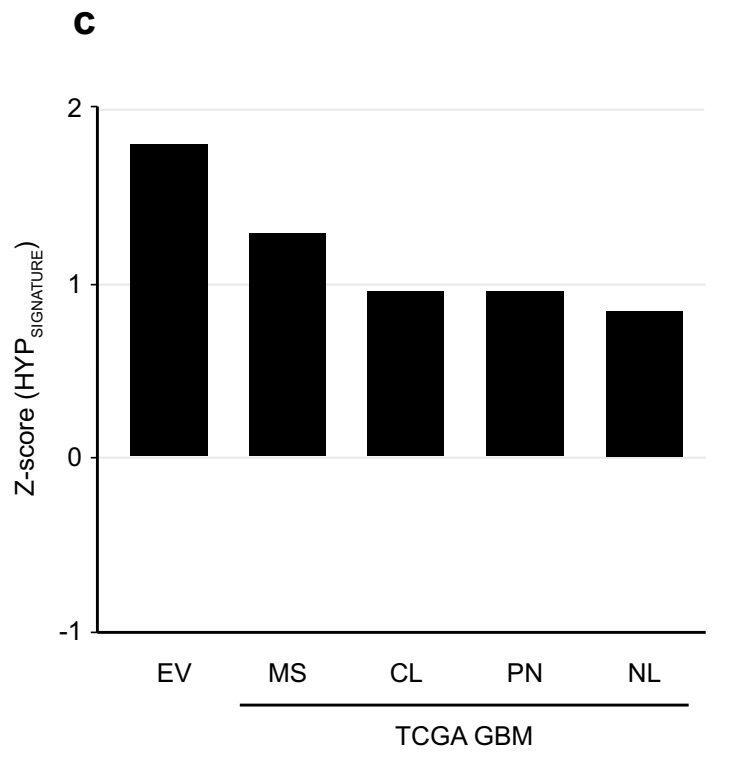

HYP $_{\text {SIGNATURE }}$ candidate genes $(N=9)$ was used as reference value for $Z$ score calculations. Bar graph showing relative HYP SIGNATURE $_{\text {S }}$ cumulative $Z$ score comparison between U87-MG cell EVs and HGCC GBM subtypes (b), and TCGA GBM subtypes (c) respectively. $M S$ mesenchymal, $C L$ classic, $P N$ proneural, and $N L$ neural 


\section{Discussion}

In this study, we used an optimized combination of nontargeted and targeted quantitative proteomics to comprehensively profile hypoxia-regulated proteins associated with high-grade glioma cell derived EVs. We have identified a protein signature, "HYP SIGNATURE", in EVs secreted by U87-MG cells that is associated with the HIF hypoxic signaling response and exhibited close proximity to the mesenchymal GBM subtype. Importantly, out of the nine proteins encompassing the $\mathrm{HYP}_{\text {SIGNATURE }}$, seven proteins are known as plasma membrane integrated proteins with an extracellular domain available for specific recognition by antibodies and other targeting agents. Together, our findings thus propose that the hypoxic status of GBM tumors can be defined by the EV HYP SIGNATURE, which may be utilized not only to noninvasively immunephenotype glioma tumors but also as potential therapeutic targets.

The utility of EVs across diverse cellular functions, including recent investigations that support the application of EVs as non-invasive biomarker tools [14, 16, 37, 38], strongly motivates improved efforts to comprehensively profile the proteome of EVs derived from cells grown at disease mimicking conditions. Using discovery proteomics, a previous study [39] identified a total of 844 proteins in EVs isolated from GBM cells. In comparison, we identified approximately 3000 proteins in EVs, out of which 1034 proteins were unique to hypoxic EVs. Importantly, the major aim of the present study was to specifically identify an EV signature that mimics the hypoxic situation, i.e. a pathognomonic feature of GBM tumors associated with disease aggressiveness and treatment resistance. Although the studies are limited to one glioma cell-line, it may be argued that the obtained results have general relevance given the substantial overlap between EV protein identities found here and the ExoCarta EV proteome database. Moreover, the hypoxic response is a universal phenomenon of high-grade gliomas as well as other highly malignant tumors. Clearly, future studies will have to further assess the generalizability of the present data, including validation in primary GBM cell models as well as in vivo.

LFQ has now become a widely accepted analytical approach for comparison of the relative abundance of proteins across multiple samples [40-42]. The possibility to analyse untreated proteins or peptides in a large number of samples makes LFQ a preferred protocol over other relative quantification approaches. However, previous studies have shown that sample preparation for the LFQ approach is highly susceptible to variability [43]. Therefore, to reduce this variability, we used 9 replicates of normoxia and 12 replicates of hypoxia samples for LFQ.
In addition, the conforming pattern of differential levels of most proteins analyzed in LFQ (Supplementary Table 6) and PRM (Supplementary Table 8), suggest a high degree of sample preparation consistency. In support of EV proteomics data, immunoblotting showed an enrichment of top candidates of the $\mathrm{HYP}_{\text {SIGNATURE}}$, and gene array analysis showed increased expression of IGFBP3, F3, CA9, SLC2A1 and PGRMC1 mRNA in hypoxic as compared with normoxic U87-MG cells. We were unable to detect other candidate proteins (NCL, SLC16A1, SPP1, ANXA5) in EVs by immunoblotting analysis, either from normoxia or hypoxia, and did not detect a hypoxic enrichment of these proteins in U87-MG cells. A potential explanation to the discrepancy between an induction of these proteins in EVs collected over a cumulative time period of $48 \mathrm{~h}$ of hypoxia, and cells analyzed at a fixed time-point, is the well-known temporal dynamics of the hypoxic response.

Several previous studies have associated tumor cell expression of HYP SIGNATURE $_{\text {proteins with increased }}$ GBM aggressiveness. For example, F3 expression was demonstrated to be hypoxia-dependent in highly aggressive P7 GBM cells, leading to increased F3 activity [44], and F3-positive EVs were shown to induce angiogenesis [20]. Hypoxia also induced increased SLC16A1 plasma membrane expression in glioma cells, both in in vitro and in vivo models [45]. Additionally, SLC16A1 plasma membrane expression was associated with HIF- $1 \alpha$ and CA9 positivity in hypoxic regions. Further, SLC16A1 was found to be upregulated in GBM as compared with normal tissues [46]. NCL was also found to be overexpressed in patient-derived GBM tumors and cells as compared with normal brain [47]. ANXA5 has been found to promote invasion and chemoresistance to the alkylating drug temozolomide in GBM cells [48]. Since hypoxic cells and components in the hypoxic niche have been increasingly implicated in resistance to temozolomide [49], it is conceivable that ANXA5 is associated with the hypoxic component of drug resistance. SPP1 was shown to be induced by hypoxia both in vitro and in vivo [50] and is predominantly observed in the microvasculature of GBM [51]. Several studies have implicated SPP1 with crucial roles in invasion [52] and malignant gliomas [53]. In several glioma cell models, CA9 strongly co-localized with HIF- $1 \alpha$, indicating its induction in hypoxic regions of this tumor type. Clinically, CA9 is minimally expressed in normal brain tissue, whereas its high expression in brain tumors strongly correlated with the level of malignancy [54]. SLC2A1 is another well-established hypoxia-induced protein that has been associated with hypoxic regions of GBM [55]. These studies support a functional role of $\mathrm{HYP}_{\text {SIGNATURE }}$ protein expression in tumor cells, and future studies that define the tumor promoting role of these proteins when associated with EVs, especially in the context of e.g. $\mathrm{pH}$ regulation 
(CA9), metabolite transport (SLC2A1, SLC16A1), and coagulation activation (F3), will be of high interest.

To conclude, our data strongly support that a specific subset of mostly membrane intercalated EV proteins could define the hypoxic status of high-grade glioma cells. The proteins identified as part of the $\mathrm{HYP}_{\text {SIGNATURE }}$ warrant further clinical examination using a targeted approach to validate their capacity to differentiate the highly heterogeneous nature of high-grade glioma tumors from e.g. low grade gliomas and other brain lesions that are challenging to define by imaging alone. This proof-of-principle study to noninvasively define the glioma hypoxic status utilizing advanced proteomics is a significant step in this direction.

\section{Materials and methods}

\section{Cells}

U87-MG cells were newly purchased from ATCC. Cells were routinely cultured in DMEM medium, supplemented with $10 \%$ foetal bovine serum (FBS), $2 \mathrm{mM} \mathrm{L}$-glutamine, $100 \mathrm{U} / \mathrm{mL}$ penicillin and $100 \mu \mathrm{g} / \mathrm{mL}$ streptomycin (growth medium). All cells were grown in humidified $5 \% \mathrm{CO}_{2}$ incubator at $37{ }^{\circ} \mathrm{C}$. For hypoxia experiments, cells were incubated in humidified Sci-tive NN Hypoxia workstation (Ruskinn Technology) set at $5 \% \mathrm{CO}_{2}, 1 \% \mathrm{O}_{2}$, and $37^{\circ} \mathrm{C}$.

\section{EV isolation}

Normoxic or hypoxic EVs were isolated in parallel from U87-MG cells at a particular passage by standard procedures, using differential ultracentrifugation [20]. Routinely cultured U87-MG cells at sub-confluency were grown in DMEM supplemented with $1 \%$ BSA at normoxic or hypoxic conditions for $48 \mathrm{~h}$. Conditioned media were collected after $48 \mathrm{~h}$ and centrifuged at $300 \times g$ twice to eliminate cell debris. Supernatant fractions were then centrifuged at $100,000 \times g$ for $2 \mathrm{~h}$ to pellet EVs, followed by washing twice with PBS at $100,000 \times g$ for $2 \mathrm{~h}$. EVs were then resuspended in $6 \mathrm{M}$ Urea for downstream proteomics experiments.

\section{Immunoblotting}

U87-MG cells or EV protein lysate were mixed with NuPAGE $4 \times$ LDS Sample Buffer (Life Technologies) and heated for $10 \mathrm{~min}$ at $80{ }^{\circ} \mathrm{C}$. Equal amount of proteins was resolved in a NuPage 4-12\% Bis Tris gel (Life Technologies) at non-reducing or reducing conditions and then transferred onto a polyvinylidene fluoride (PVDF) membrane (Immobilon-FL), followed by blocking in TBS containing $0.05 \%$ Tween 20,5\% nonfat dry milk or 3\% BSA for $1 \mathrm{~h}$ at RT. To probe for CD9, CD63, CD81, Flotillin-1, IGFBP3, and
CA9, the membrane was incubated with the following antibodies in TBST containing 5\% nonfat dry milk overnight at $4{ }^{\circ} \mathrm{C}$ : anti-CD9 (1:2000; ab92726, Abcam), anti-CD63 (1:100; ab8219, Abcam), anti-CD81 (1:1000; ab109201, Abcam), anti-flotillin-1 (1 $\mu \mathrm{g} / \mathrm{mL}$; ab41927, Abcam), Rabbit anti-IGFBP3 (1:80; PAAJ1, GroPep), M75 anti-CA9 (1:300; M75, Bioscience Slovakia), Mouse anti-NCL (1:1000, ab13541, Abcam), Rabbit anti-SLC16A1 (1:1000, ab179832, Abcam), Mouse anti-SPP1 (1:500, ab166709, Abcam), and Rabbit anti-ANXA5 (1:500, ab14196, Abcam). After washing, the membrane was incubated with HRPconjugated anti-mouse IgG $(1: 10,000)$ (A9044, SigmaAldrich) or anti-rabbit secondary antibody (1:3000) (7074, Cell Signaling Technology). Protein bands were visualized by enhanced chemiluminescence western blotting substrate (Pierce).

Nanoparticle Tracking Analysis, Transmission Electron Microscopy, Trypsin digestion and peptide preparation, Discovery LC-MS/MS, label free quantification, and quantitative LC-PRM-MS/MS were performed as described in Supplementary Materials and Methods.

\section{Data analysis}

The Gene Ontology functional classification of $\mathrm{H}_{\text {significant }}$ proteins was performed using PANTHER (https://www. pantherdb.org/). Enriched pathways of $\mathrm{EV}_{\mathrm{HYP}}$ signature proteins were determined using ConsensusPathDB-human interaction database (https://cpdb.molgen.mpg.de/). Wilcoxon test was employed for pathway enrichment analysis with a $P$ value cut-off of 0.01 .

Gene expression data on different GBM subtypes were obtained from The Cancer Genome Atlas (TCGA) via the GlioVis portal (https://gliovis.bioinfo.cnio.es/), as well as from the Human Glioma Cell Cultures (HGCC) database (https://www.hgcc.se/).

For HYP SIGNATURE $_{\text {comparison in U87-MG cell-derived }}$ EVs, the $Z$ scores of $9 \mathrm{HYP}_{\text {SIGNATURE }}$ candidates were individually calculated for their protein levels with the respective normoxic values as reference as shown by the formula below:

Z-score $=\left(\mathrm{EV}_{\mathrm{HYP}}-\mathrm{EV}_{\text {NORM }}\right) /\left(\mathrm{SD} \mathrm{EV}_{\text {NORM }}\right)$

where " $E V_{\text {HYP" }}$ is the mean protein level measured in hypoxic EVs; " $\mathrm{EV}_{\mathrm{NORM}}$ " is the mean protein level meas-

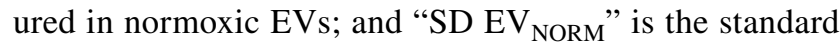
deviation value of the protein level measurements in normoxic EVs. Generation of a cumulative score was done by arithmetic mean of $Z$ scores of all $9 \mathrm{HYP}_{\text {SIGNATURE }}$ proteins.

For $Z$ score calculation on the TCGA dataset, subtype classification of GBM patients was performed with GlioVis portal, and gene expression values for all $9 \mathrm{HYP}_{\text {SIGNATURE }}$ candidates were downloaded. Low Grade Glioma (LGG) 
expression data of the $9 \mathrm{HYP}_{\text {SIGNATURE }}$ protein genes was downloaded and used as reference value for $Z$ score calculations, as indicated in the formula below:

Z-score $=($ GBM subtype-TCGA - LGG $) /($ SD TCGA - LGG $)$ where "GBM subtype" is the mean gene expression value in subtypes such as Classical, Mesenchymal, or Proneural GBM; "TCGA-LGG" is the mean gene expression value for the corresponding gene in LGG patients; and "SD TCGALGG" is the standard deviation value of the analyzed gene among the LGG patients. Generation of cumulative score for each GBM subtype was done by arithmetic mean of $Z$ scores of all 9 HYP $_{\text {SIGNATURE }}$ candidates.

For HGCC data analysis, the gene expression $Z$ score for each HYP ${ }_{\text {SIGNATURE }}$ candidate in subtypes (Classical, Mesenchymal, Proneural, or Neural) was directly extracted from the HGCC database. Cumulative $Z$ score was generated as described for TCGA dataset.

\section{Statistical analyses}

Data are expressed as mean \pm STDEV. Statistical analyses were done using unpaired Student $t$ test. All values with $P<0.05$ were considered to be statistically significant.

Acknowledgements Open access funding provided by Lund University. We thank Maria Johansson and Melinda Rezeli for their advice with parallel reaction monitoring protocol optimization. This study was funded by grants (to MB) from the Swedish Cancer Fund; the Swedish Research Council; the Swedish Childhood Cancer Foundation; the Gunnar Nilsson Cancer Foundation; the Mrs Berta Kamprad Foundations; the Skåne University Hospital donation funds; the Governmental funding of clinical research within the national health services, ALF; and a donation by Viveca Jeppsson. Strategic infrastructure support from ThermoFisher Scientific (to GMV) is also acknowledged.

\section{Compliance with ethical standards}

Conflict of interest The authors declare that they have no conflicts of interest.

Open Access This article is distributed under the terms of the Creative Commons Attribution 4.0 International License (http://creativeco mmons.org/licenses/by/4.0/), which permits unrestricted use, distribution, and reproduction in any medium, provided you give appropriate credit to the original author(s) and the source, provide a link to the Creative Commons license, and indicate if changes were made.

\section{References}

1. Schwartzbaum JA, Fisher JL, Aldape KD, Wrensch M (2006) Epidemiology and molecular pathology of glioma. Nat Clin Pract Neurol 2:494-503

2. Stark AM, Nabavi A, Mehdorn HM, Blomer U (2005) Glioblastoma multiforme-report of 267 cases treated at a single institution. Surg Neurol 63:162-169
3. Arvold ND, Reardon DA (2014) Treatment options and outcomes for glioblastoma in the elderly patient. Clin Interv Aging 9:357-367

4. Evans SM, Judy KD, Dunphy I, Jenkins WT, Hwang WT, Nelson PT, Lustig RA, Jenkins K, Magarelli DP, Hahn SM, Collins RA, Grady MS, Koch CJ (2004) Hypoxia is important in the biology and aggression of human glial brain tumors. Clin Cancer Res 10:8177-8184

5. Cooper LAD, Gutman DA, Chisolm C, Appin C, Kong J, Rong Y, Kurc T, Van Meir EG, Saltz JH, Moreno CS, Brat DJ (2012) The tumor microenvironment strongly impacts master transcriptional regulators and gene expression class of glioblastoma. Am J Pathol 180:2108-2119

6. Masson N, Ratcliffe PJ (2014) Hypoxia signaling pathways in cancer metabolism: the importance of co-selecting interconnected physiological pathways. Cancer Metab 2:3-3

7. Rong Y, Durden DL, Van Meir EG, Brat DJ (2006) 'Pseudopalisading' necrosis in glioblastoma: a familiar morphologic feature that links vascular pathology, hypoxia, and angiogenesis. J Neuropathol Exp Neurol 65:529-539

8. Harris AL (2002) Hypoxia-a key regulatory factor in tumour growth. Nat Rev Cancer 2:38-47

9. Pouyssegur J, Dayan F, Mazure NM (2006) Hypoxia signalling in cancer and approaches to enforce tumour regression. Nature 441:437-443

10. Bertout JA, Patel SA, Simon MC (2008) The impact of $\mathrm{O}_{2}$ availability on human cancer. Nat Rev Cancer 8:967-975

11. Finger EC, Giaccia AJ (2010) Hypoxia, inflammation, and the tumor microenvironment in metastatic disease. Cancer Metastasis Rev 29:285-293

12. Schito L, Semenza GL (2016) Hypoxia-inducible factors: master regulators of cancer progression. Trends Cancer 2:758-770

13. Walsh JC, Lebedev A, Aten E, Madsen K, Marciano L, Kolb HC (2014) The clinical importance of assessing tumor hypoxia: relationship of tumor hypoxia to prognosis and therapeutic opportunities. Antioxid Redox Signal 21:1516-1554

14. Chandran VI, Welinder C, Mansson AS, Offer S, Freyhult E, Pernemalm M, Lund SM, Pedersen S, Lehtio J, Marko-Varga G, Johansson MC, Englund EM, Sundgren PC, Belting M (2019) Ultrasensitive immunoprofiling of plasma extracellular vesicles identifies syndecan-1 as a potential tool for minimally invasive diagnosis of glioma. Clin Cancer Res. https://doi. org/10.1158/1078-0432.CCR-18-2946.

15. Xu R, Rai A, Chen M, Suwakulsiri W, Greening DW, Simpson RJ (2018) Extracellular vesicles in cancer-implications for future improvements in cancer care. Nat Rev Clin Oncol 15:617-638

16. Melo SA, Luecke LB, Kahlert C, Fernandez AF, Gammon ST, Kaye J, LeBleu VS, Mittendorf EA, Weitz J, Rahbari N, Reissfelder C, Pilarsky C, Fraga MF, Piwnica-Worms D, Kalluri R (2015) Glypican-1 identifies cancer exosomes and detects early pancreatic cancer. Nature 523:177-182

17. Yang Y, Hong Y, Cho E, Kim GB, Kim I-S (2018) Extracellular vesicles as a platform for membrane-associated therapeutic protein delivery. J Extracell Vesicles 7:1440131-1440131

18. Lobb RJ, Becker M, Wen SW, Wong CS, Wiegmans AP, Leimgruber A, Moller A (2015) Optimized exosome isolation protocol for cell culture supernatant and human plasma. J Extracell Vesicles 4:27031

19. Bang-Rudenstam A, Cerezo-Magana M, and Belting M (2019) Pro-metastatic functions of lipoproteins and extracellular vesicles in the acidic tumor microenvironment. Cancer Metastasis Rev

20. Svensson KJ, Kucharzewska P, Christianson HC, Skold S, Lofstedt T, Johansson MC, Morgelin M, Bengzon J, Ruf W, Belting M (2011) Hypoxia triggers a proangiogenic pathway involving cancer cell microvesicles and PAR-2-mediated heparin-binding 
EGF signaling in endothelial cells. Proc Natl Acad Sci USA 108:13147-13152

21. Drucker KL, Kitange GJ, Kollmeyer TM, Law ME, Passe S, Rynearson AL, Blair H, Soderberg CL, Morlan BW, Ballman KV, Giannini C, Jenkins RB (2009) Characterization and gene expression profiling in glioma cell lines with deletion of chromosome 19 before and after microcell-mediated restoration of normal human chromosome 19. Genes Chromosomes Cancer 48:854-864

22. Jung T-Y, Choi Y-D, Kim Y-H, Lee J-J, Kim H-S, Kim J-S, Kim S-K, Jung S, Cho D (2013) Immunological characterization of glioblastoma cells for immunotherapy. Anticancer Res 33:2525-2533

23. Böing AN, van der Pol E, Grootemaat AE, Coumans FAW, Sturk A, Nieuwland R (2014) Single-step isolation of extracellular vesicles by size-exclusion chromatography. J Extracell Vesicles. https ://doi.org/10.3402/jev.v3.23430

24. Kucharzewska P, Belting M (2013) Emerging roles of extracellular vesicles in the adaptive response of tumour cells to microenvironmental stress. J Extracell Vesicles. https://doi.org/10.3402/jev. v2i0.20304

25. Shao C, Yang F, Miao S, Liu W, Wang C, Shu Y, Shen H (2018) Role of hypoxia-induced exosomes in tumor biology. Mol Cancer 17:120

26. Raposo G, Stoorvogel W (2013) Extracellular vesicles: exosomes, microvesicles, and friends. J Cell Biol 200:373-383

27. Kowal J, Arras G, Colombo M, Jouve M, Morath JP, PrimdalBengtson B, Dingli F, Loew D, Tkach M, Thery C (2016) Proteomic comparison defines novel markers to characterize heterogeneous populations of extracellular vesicle subtypes. Proc Natl Acad Sci USA 113:E968-E977

28. Keerthikumar S, Chisanga D, Ariyaratne D, Al Saffar H, Anand S, Zhao K, Samuel M, Pathan M, Jois M, Chilamkurti N, Gangoda L, Mathivanan S (2016) ExoCarta: a web-based compendium of exosomal cargo. J Mol Biol 428:688-692

29. Mi H, Thomas P (2009) PANTHER pathway: an ontology-based pathway database coupled with data analysis tools. In: Nikolsky Y, Bryant J (eds) Protein networks and pathway analysis. Humana Press, Totowa, NJ, pp 123-140

30. DeBerardinis RJ, Chandel NS (2016) Fundamentals of cancer metabolism Science advances 2:e1600200-e1600200

31. Eales KL, Hollinshead KER, Tennant DA (2016) Hypoxia and metabolic adaptation of cancer cells. Oncogenesis 5:e190

32. Rauniyar N (2015) Parallel reaction monitoring: a targeted experiment performed using high resolution and high mass accuracy mass spectrometry. Int J Mol Sci 16:28566-28581

33. Kamburov A, Stelzl U, Lehrach H, Herwig R (2013) The ConsensusPathDB interaction database: 2013 update. Nucleic Acids Res 41:D793-D800

34. Phillips HS, Kharbanda S, Chen R, Forrest WF, Soriano RH, Wu TD, Misra A, Nigro JM, Colman H, Soroceanu L, Williams PM, Modrusan Z, Feuerstein BG, Aldape K (2006) Molecular subclasses of high-grade glioma predict prognosis, delineate a pattern of disease progression, and resemble stages in neurogenesis. Cancer Cell 9:157-173

35. Verhaak RG, Hoadley KA, Purdom E, Wang V, Qi Y, Wilkerson MD, Miller CR, Ding L, Golub T, Mesirov JP, Alexe G, Lawrence M, O'Kelly M, Tamayo P, Weir BA, Gabriel S, Winckler W, Gupta S, Jakkula L, Feiler HS, Hodgson JG, James CD, Sarkaria JN, Brennan C, Kahn A, Spellman PT, Wilson RK, Speed TP, Gray JW, Meyerson M, Getz G, Perou CM, Hayes DN (2010) Integrated genomic analysis identifies clinically relevant subtypes of glioblastoma characterized by abnormalities in PDGFRA, IDH1, EGFR, and NF1. Cancer Cell 17:98-110

36. Zarkoob H, Taube JH, Singh SK, Mani SA, Kohandel M (2013) Investigating the link between molecular subtypes of glioblastoma, epithelial-mesenchymal transition, and CD133 cell surface protein. PLoS ONE 8:e64169

37. Yang KS, Im H, Hong S, Pergolini I, Del Castillo AF, Wang R, Clardy S, Huang CH, Pille C, Ferrone S, Yang R, Castro CM, Lee H, Del Castillo CF, Weissleder R (2017) Multiparametric plasma EV profiling facilitates diagnosis of pancreatic malignancy. Sci Transl Med 9(391):eaal3226

38. Figueroa JM, Skog J, Akers J, Li H, Komotar R, Jensen R, Ringel F, Yang I, Kalkanis S, Thompson R, LoGuidice L, Berghoff E, Parsa A, Liau L, Curry W, Cahill D, Bettegowda C, Lang FF, Chiocca EA, Henson J, Kim R, Breakefield X, Chen C, Messer K, Hochberg F, Carter BS (2017) Detection of wild-type EGFR amplification and EGFRvIII mutation in CSF-derived extracellular vesicles of glioblastoma patients. Neuro Oncol 19:1494-1502

39. Mallawaaratchy DM, Hallal S, Russell B, Ly L, Ebrahimkhani S, Wei H, Christopherson RI, Buckland ME, Kaufman KL (2017) Comprehensive proteome profiling of glioblastoma-derived extracellular vesicles identifies markers for more aggressive disease. $\mathrm{J}$ Neuro-Oncol 131:233-244

40. Bondarenko PV, Chelius D, Shaler TA (2002) Identification and relative quantitation of protein mixtures by enzymatic digestion followed by capillary reversed-phase liquid chromatographytandem mass spectrometry. Anal Chem 74:4741-4749

41. Liu H, Sadygov RG, Yates JR 3rd (2004) A model for random sampling and estimation of relative protein abundance in shotgun proteomics. Anal Chem 76:4193-4201

42. Asara JM, Christofk HR, Freimark LM, Cantley LC (2008) A label-free quantification method by MS/MS TIC compared to SILAC and spectral counting in a proteomics screen. Proteomics 8:994-999

43. Bantscheff M, Lemeer S, Savitski MM, Kuster B (2012) Quantitative mass spectrometry in proteomics: critical review update from 2007 to the present. Anal Bioanal Chem 404:939-965

44. Monteiro RQ, Lima LG, Goncalves NP, De Souza MRA, Leal AC, Demasi MAA, Sogayar MC, Carneiro-Lobo TC (2016) Hypoxia regulates the expression of tissue factor pathway signaling elements in a rat glioma model. Oncol Lett 12:315-322

45. Miranda-Goncalves V, Granja S, Martinho O, Honavar M, Pojo M, Costa BM, Pires MM, Pinheiro C, Cordeiro M, Bebiano G, Costa P, Reis RM, Baltazar F (2016) Hypoxia-mediated upregulation of MCT1 expression supports the glycolytic phenotype of glioblastomas. Oncotarget 7:46335-46353

46. Miranda-Goncalves V, Honavar M, Pinheiro C, Martinho O, Pires MM, Pinheiro C, Cordeiro M, Bebiano G, Costa P, Palmeirim I, Reis RM, Baltazar F (2013) Monocarboxylate transporters (MCTs) in gliomas: expression and exploitation as therapeutic targets. Neuro Oncol 15:172-188

47. Balça-Silva J, do Carmo A, Tão H, Rebelo O, Barbosa M, MouraNeto V, Sarmento-Ribeiro AB, Lopes MC, Moreira JN (2018) Nucleolin is expressed in patient-derived samples and glioblastoma cells, enabling improved intracellular drug delivery and cytotoxicity. Exp Cell Res 370:68-77

48. Wu L, Yang L, Xiong Y, Guo H, Shen X, Cheng Z, Zhang Y, Gao Z, Zhu X (2014) Annexin A5 promotes invasion and chemoresistance to temozolomide in glioblastoma multiforme cells. Tumor Biol 35:12327-12337

49. Ge X, Pan M-H, Wang L, Li W, Jiang C, He J, Abouzid K, Liu L-Z, Shi Z, Jiang B-H (2018) Hypoxia-mediated mitochondria apoptosis inhibition induces temozolomide treatment resistance through miR-26a/Bad/Bax axis. Cell Death Dis 9:1128-1128

50. Said HM, Hagemann C, Staab A, Stojic J, Kuhnel S, Vince GH, Flentje M, Roosen K, Vordermark D (2007) Expression patterns of the hypoxia-related genes osteopontin, CA9, erythropoietin, VEGF and HIF-1alpha in human glioma in vitro and in vivo. Radiother Oncol 83:398-405 
51. Takano S, Tsuboi K, Tomono Y, Mitsui Y, Nose T (2000) Tissue factor, osteopontin, alphavbeta3 integrin expression in microvasculature of gliomas associated with vascular endothelial growth factor expression. Br J Cancer 82:1967-1973

52. Colin C, Baeza N, Bartoli C, Fina F, Eudes N, Nanni I, Martin PM, Ouafik L, Figarella-Branger D (2006) Identification of genes differentially expressed in glioblastoma versus pilocytic astrocytoma using Suppression Subtractive Hybridization. Oncogene 25:2818-2826

53. Jan H-J, Lee C-C, Shih Y-L, Hueng D-Y, Ma H-I, Lai J-H, Wei H-W, Lee H-M (2010) Osteopontin regulates human glioma cell invasiveness and tumor growth in mice. Neuro Oncol 12:58-70

54. Proescholdt MA, Mayer C, Kubitza M, Schubert T, Liao S-Y, Stanbridge EJ, Ivanov S, Oldfield EH, Brawanski A, Merrill MJ
(2005) Expression of hypoxia-inducible carbonic anhydrases in brain tumors. Neuro Oncol 7:465-475

55. Joseph JV, Conroy S, Pavlov K, Sontakke P, Tomar T, EggensMeijer E, Balasubramaniyan V, Wagemakers M, den Dunnen WFA, Kruyt FAE (2015) Hypoxia enhances migration and invasion in glioblastoma by promoting a mesenchymal shift mediated by the HIF1 $\alpha-Z E B 1$ axis. Cancer Lett 359:107-116

Publisher's Note Springer Nature remains neutral with regard to jurisdictional claims in published maps and institutional affiliations. 